\title{
Structural Relaxation and Refractive Index of Low-Loss Poly(methyl methacrylate) Glass
}

\author{
Norihisa TANIO \\ Faculty of Photonics Science and Technology, Chitose Institute of Science and Technology, \\ 758-65 Bibi, Chitose 066-8655, Japan
}

(Received November 1, 2001; Accepted April 9, 2002)

\begin{abstract}
KEY WORDS Structural Relaxation / Physical Aging / Refractive Index / Prism Coupling / Light Scattering Loss / Glass Transition Temperature / Poly(methyl methacrylate) /
\end{abstract}

Amorphous polymer glasses are essentially in thermodynamic non-equilibrium states. Physical aging ${ }^{1}$ describes the time dependence of change in the behavior of a polymer glass held at temperatures below the glass transition. Such change is normally the result of continuous slow relaxation of the glass from its initial non-equilibrium state towards the final thermodynamic equilibrium state. Thermodynamical properties such as volume $^{2,3}$ or enthalpy ${ }^{4,5}$ have been mainly considered for the study of this phenomenon. Many properties of amorphous polymer glasses are affected by aging. Recently, we investigated the effects of enthalpy relaxation on light scattering loss of low-loss Poly(methylmethacrylate) (PMMA) glass which had no excess scattering, and concluded that light scattering loss of glassy PMMA is not affected inherently by physical aging due to approach to thermodynamic equilibrium at a temperature below glass transition temperature $\left(T_{\mathrm{g}}\right)^{6}$

In this paper, the volume relaxation of low-loss PMMA glass was studied by using optical prism coupling method. We measured the change of refractive indices of low-loss PMMA glass with annealing at temperatures below $T_{\mathrm{g}}$, and tried to determine the volume relaxation by physical aging.

\section{EXPERIMENTAL}

\section{Preparation of PMMA Glass}

After ordinary purification of methyl methacrylate (MMA) monomer with a distillation (bp 46$47^{\circ} \mathrm{C} / 100 \mathrm{mmHg}$ ) and $0.2 \mu \mathrm{m}$ membrane filter, rigorous purification was carried out as follows: Ampoule A with distilled monomer was connected with two ampoules B and C carefully purified. Here, di-tert-butyl peroxide (DBPO) as an initiator and $n$-butyl mercaptan $(n \mathrm{BM})$ as a chain transfer agent were placed in ampoule
$\mathrm{B}$, and ampoule $\mathrm{C}$ was empty. Ampoules $\mathrm{A}$ and $\mathrm{B}$ were frozen with liquid nitrogen, evacuated, and substituted by nitrogen. The monomers, $\mathrm{DBPO}$, and $n \mathrm{BM}$ were degassed by several freeze-thaw cycles, and slowly distilled into the ampoule $\mathrm{C}$ under vacuum by cooling ampoule $\mathrm{C}$ with liquid nitrogen. Ampoule $\mathrm{C}$ was sealed under vacuum and immersed in silicone oil for polymerization. After polymerization, the cylindrical polymer sample with a $20 \mathrm{~mm}$ diameter was removed from ampoule $\mathrm{C}$ for measurement.

\section{Enthalpy Relaxation Measurement}

Enthalpy relaxation of polymer glasses was measured by DSC (Shimadzu Co., DSC-50). The following standard thermal history was employed for the low-loss PMMA sample:

1) To eliminate the effects of previous thermal history after polymerization, the PMMA sample was annealed at $110^{\circ} \mathrm{C}$ for $3 \mathrm{~h}$.

2) The PMMA sample was annealed for an aging time of $t_{\mathrm{a}}$ at chosen aging temperatures $\left(T_{\mathrm{a}}\right)$.

3) The PMMA sample was quenched down to the room temperature.

4) DSC measurements were carried out with increasing temperature from room temperature to $170^{\circ} \mathrm{C}$ at a heating rate of $10^{\circ} \mathrm{C} \mathrm{min}-1$.

\section{Light Scattering Measurement}

Light scattering measurements were made with light scattering spectrophotometer (Otsuka Electronics, DLS-7000). The details of theory and analytical procedure of light scattering are described elsewhere. ${ }^{7}$ Polarized $\left(V_{\mathrm{V}}\right)$ and depolarized $\left(H_{\mathrm{V}}\right)$ scattered intensities were observed at the incident wavelength $\lambda_{0}=633 \mathrm{~nm}$. The letters $H$ and $V$ (or $\mathrm{v}_{\mathrm{V}}$ ) denote horizontal and vertical polarization, respectively, and the upper case letter 
and subscript denote the scattered and incident beam, respectively.

To analyze the local structure, we separated $V_{\mathrm{V}}$ into the two terms of eq 1 .

$$
V_{\mathrm{V}}=V_{\mathrm{V} 1}+V_{\mathrm{V} 2}
$$

where $V_{\mathrm{V} 1}$ denotes the background intensity independent of the scattering angle, and $V_{\mathrm{V} 2}$ denotes the excess scattering with angular dependence due to large size heterogeneities. In a structureless liquid or randomly oriented polymer bulk, the isotropic part $V_{\mathrm{V} 1}$ iso of $V_{\mathrm{V} 1}$ is given by eq 2 .

$$
V_{\mathrm{V} 1}{ }^{\text {iso }}=V_{\mathrm{V} 1}-(4 / 3) H_{\mathrm{V}}
$$

$H_{\mathrm{V}}$ intensity for our sample examined in this paper had no angular dependence.

Scattering loss was estimated by integrating scattering intensity in all directions. Total light scattering loss $\alpha_{\mathrm{t}}\left(\mathrm{dB} \mathrm{km}^{-1}\right)$ is composed of three terms, i.e., $\alpha_{1}$ iso, $\alpha_{2}{ }^{\text {iso }}$, and $\alpha^{\text {aniso }} . \alpha_{1}{ }^{\text {iso }}$ is loss due to isotropic $V_{\mathrm{V} 1}{ }^{\text {iso }}$ scattering without angular dependence, $\alpha_{2}{ }^{\text {iso }}$ is due to isotropic $V_{\mathrm{V} 2}$ scattering with angular dependence, and $\alpha^{\text {aniso }}$ is due to anisotropic scattering, $H_{\mathrm{V}}$.

$$
\begin{gathered}
\alpha_{1}{ }^{\text {iso }}\left(\mathrm{dB} \mathrm{km}^{-1}\right)=1.16 \times 10^{6} \pi V_{\mathrm{V} 1} \text { iso } \\
\alpha_{2}{ }^{\text {iso }}\left(\mathrm{dB} \mathrm{km}^{-1}\right)=\frac{1.35 \times 10^{9} a^{3}<\eta^{2}>}{n^{4} \lambda^{4}} \\
\times\left[\frac{(b+2)^{2}}{b^{2}(b+1)}-\frac{2(b+2)}{b^{3}} \ln (b+1)\right] \\
b=\frac{16 \pi^{2} a^{2}}{\lambda^{2}} \\
\alpha^{\text {aniso }}\left(\mathrm{dB} \mathrm{km}^{-1}\right)=3.86 \times 10^{6} \pi H_{\mathrm{V}}
\end{gathered}
$$

Equation 4 is obtained using Debye's theory. ${ }^{8,9} \mathrm{Sym}-$ bol $a(\AA)$ is correlation length and a measure of the size of the heterogeneous structure inside the bulk. Symbol $<\eta^{2}>$ denotes the mean-square average of fluctuation of all dielectric constants, $n$ is the refractive index of medium, and $\lambda$ is the wavelength of light in a medium.

\section{Refractive Index Measurement}

Refractive indices of PMMA glasses were determined with prism coupler (Metricon model 2010 prism coupler). Prism coupling ${ }^{10,11}$ is convenient and refractive index change as small as $10^{-4}$ can be measured. A TE polarized He-Ne laser (wavelength $633 \mathrm{~nm}$ ) was used to monitor the refractive indices.

\section{Density Measurements}

Density was compared by preparing aqueous solutions of calcium nitrate and determining whether test specimens sank or floated in these standard solutions. ${ }^{12}$

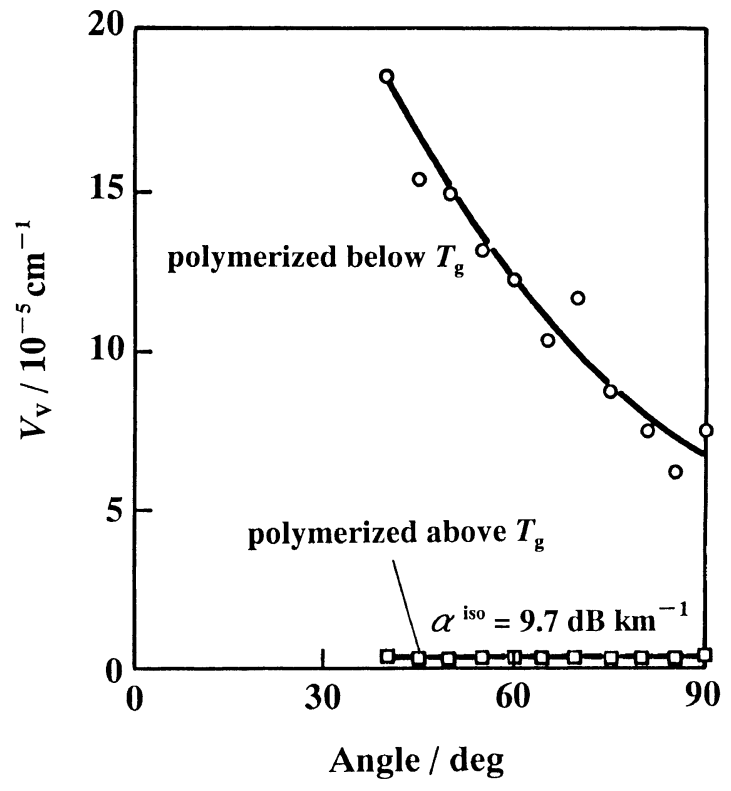

Figure 1. $V_{\mathrm{V}}$ scattering by PMMA glasses polymerized at $70^{\circ} \mathrm{C}$ for $216 \mathrm{~h}(\mathrm{O})$, and $130^{\circ} \mathrm{C}$ for $96 \mathrm{~h}(\square)$.

\section{RESULTS AND DISCUSSION}

Light scattering loss of PMMA glasses, even in using contaminant-free monomer varied widely with polymerization and heat-treatment conditions. ${ }^{7,13-15}$ Figure 1 shows the isotropic light scattering intensity $\left(V_{\mathrm{V}}\right)$ at $633 \mathrm{~nm}$ for two PMMA glasses. One was polymerized at $70^{\circ} \mathrm{C}$ (below the $T_{\mathrm{g}}$ ) for $216 \mathrm{~h}$. The other was polymerized at $130^{\circ} \mathrm{C}$ (above the $T_{\mathrm{g}}$ ) for $96 \mathrm{~h}$. With polymerization at $70^{\circ} \mathrm{C}$, angular dependence of $V_{\mathrm{V}}$ intensity was observed. The angular dependence of light scattering intensity suggested the existence of heterogeneous structure ( $c a .860 \AA$ ) with the order of $10^{-4}$ of refractive-index differences. The total scattering loss of the PMMA glass was $325 \mathrm{~dB} \mathrm{~km}^{-1}$ at $633 \mathrm{~nm}$. With polymerization at $130^{\circ} \mathrm{C}$, no angular dependence was observed and isotropic light scattering loss $\left(\alpha^{\text {iso }}\right)$ was reduced to $9.7 \mathrm{~dB} \mathrm{~km}^{-1}$ (at $633 \mathrm{~nm}$ ) which is close to the value predicted by the thermally induced fluctuation theory.

Using the fluctuation theory, ${ }^{16}$ the intensity of the isotropic light scattering $\left(V_{\mathrm{V}}{ }^{\text {iso }}\right)$ from thermally induced density fluctuations in a structureless liquid is

$$
V_{\mathrm{V}}^{\text {iso }}=\frac{\pi^{2}}{9 \lambda_{0}{ }^{4}}\left(n^{2}-1\right)^{2}\left(n^{2}+2\right)^{2} k T \beta
$$

where $\lambda_{0}$ is the wavelength of light in a vacuum, $k$ is the Boltzmann constant, $T$ is the absolute temperature, $n$ is the refractive index and $\beta$, isothermal compressibility. The fluctuation theory for structureless liquids indicates that according to eq 6 , the isotropic scattering loss decreases with decrease in isothermal compressibility and 


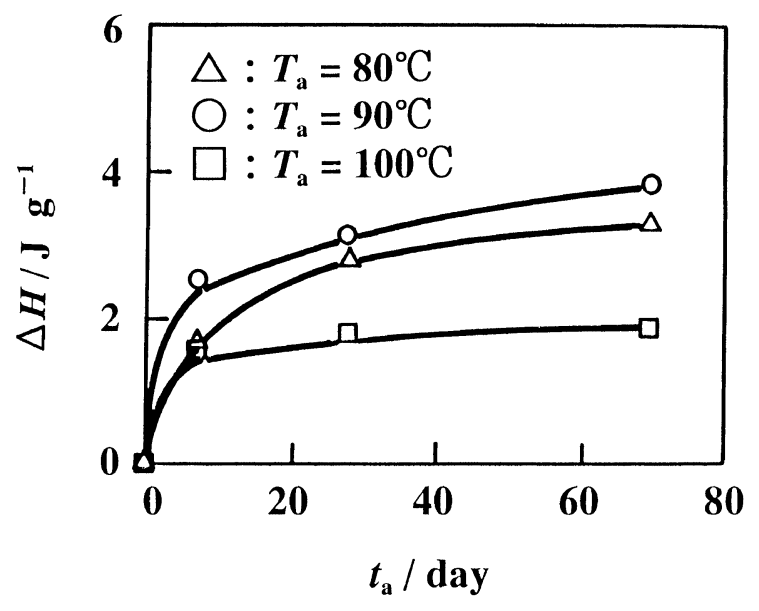

Figure 2. Enthalpy relaxation $(\Delta H)$ plotted as a function of aging time $\left(t_{\mathrm{a}}\right)$ at various aging temperature $\left(T_{\mathrm{a}}\right)$ for low-loss PMMA glass.

refractive index. $V_{\mathrm{V}}$ iso calculated using the value of $\beta$ at $T_{\mathrm{g}}$ according to a frozen model showed good agreement with that observed for PMMA glass. When the reported $\beta$ at $100^{\circ} \mathrm{C}$ (near $T_{\mathrm{g}}$ ) is used in eq 6 , the calculated $\alpha^{\text {iso }}$ is $9.5 \mathrm{~dB} \mathrm{~km}^{-1}$ which is close to our observed value $\left(9.7 \mathrm{~dB} \mathrm{~km}^{-1}\right)$ for PMMA glass polymerized above the $T_{\mathrm{g}}$ in Figure 1. In this study, low-loss PMMA glass (9.7 $\mathrm{dB} \mathrm{km} \mathrm{k}^{-1}$ at $633 \mathrm{~nm}$ ) with no excess scattering was used for measurements. This PMMA sample was polymerized at $130^{\circ} \mathrm{C}$ for $96 \mathrm{~h}$ and the weight percentages of DBPO and $n$ BM were each $0.20 \mathrm{wt} \%$.

Heat capacity of a polymer glass undergoes a step change in the glass transition region. We define the glass transition temperature, $T_{\mathrm{g}}$, as the temperature at which the change of heat capacity associated with glass transition is $\Delta C_{\mathrm{p}} / 2$, where $\Delta C_{\mathrm{p}}$ is the specific heat increment between the liquid and glass states at glass transition region. $T_{\mathrm{g}}$ for unaged low-loss PMMA glass was $108^{\circ} \mathrm{C}$.

Physical aging is time- and temperature-dependent. ${ }^{17}$ Most studies on physical aging for amorphous polymers have been performed at temperatures close to $T_{\mathrm{g}}$. We measured the enthalpy relaxation for lowloss PMMA glass at aging temperatures, $80^{\circ} \mathrm{C}\left(=T_{\mathrm{g}}-\right.$ $\left.28^{\circ} \mathrm{C}\right), 90^{\circ} \mathrm{C}\left(=T_{\mathrm{g}}-18^{\circ} \mathrm{C}\right)$, and $100^{\circ} \mathrm{C}\left(=T_{\mathrm{g}}-8^{\circ} \mathrm{C}\right)$. Enthalpy relaxation $\Delta H$ can be determined by taking the difference between the DSC curve corresponding to the quenched state and curves corresponding to different aged states. Figure 2 presents evolution of $\Delta H$ as a function of aging time at various $T_{\mathrm{a}}$ for low-loss PMMA glass. With increasing aging time, enthalpy relaxation increased, and reached $3.3,3.8$, and $1.8 \mathrm{~J} \mathrm{~g}^{-1}$, at $T_{\mathrm{a}}=80^{\circ} \mathrm{C}, 90^{\circ} \mathrm{C}$, and $100^{\circ} \mathrm{C}$, respectively. Maximum enthalpy relaxation was $90^{\circ} \mathrm{C}\left(=T_{\mathrm{g}}-18^{\circ} \mathrm{C}\right)$.

Refractive index measurements were performed with prism-coupler down to room temperature after isother-

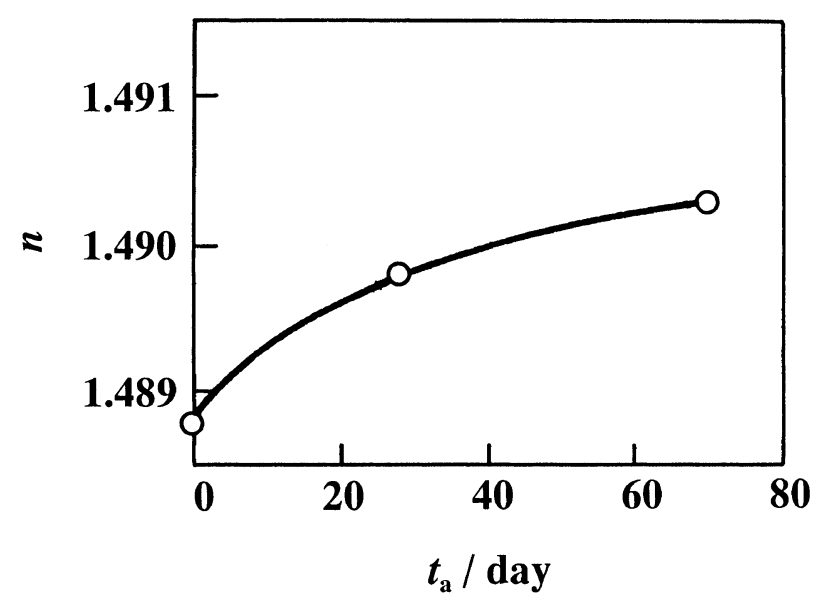

Figure 3. Refractive index $(n)$ at $633 \mathrm{~nm}$ plotted as a function of aging time $\left(t_{\mathrm{a}}\right)$ at $90^{\circ} \mathrm{C}$ for low-loss PMMA glass.

mal aging at $90^{\circ} \mathrm{C}$. Figure 3 shows the refractive index $\left(n_{\mathrm{TE}}\right)$ at $633 \mathrm{~nm}$ for low-loss PMMA, as function of aging time at $90^{\circ} \mathrm{C}$. Before aging, $n_{\mathrm{TE}}$ of PMMA was 1.4888 . With increasing aging time, $n_{\mathrm{TE}}$ slightly increased, reaching to 1.4903 at 70 days. The refractive index change $(\Delta n)$ by aging at $90^{\circ} \mathrm{C}$ for 70 days is 0.0015 . It is considered that volume relaxation occurs with isothermal aging, and density increases, and refractive index would increase as a result. Density measurement for low-loss PMMA glass was performed with isothermal aging at $90^{\circ} \mathrm{C}$. Before aging, density was $1.191 \mathrm{~g} \mathrm{~cm}^{-3}$. With increasing time, density increased, and reached $1.193 \mathrm{~g} \mathrm{~cm}^{-3}$ at 70 days. The change of density $(\Delta \rho)$ by aging at $90^{\circ} \mathrm{C}$ for 70 days is $0.002 \mathrm{~g} \mathrm{~cm}^{-3}$.

The refractive index $(n)$ of a compound can be calculated from its molar refraction and molecular volume using the Lorentz-Lorenz equation, ${ }^{18,19}$

$$
n=\sqrt{\left(2 \frac{[R]}{V}+1\right) /\left(1-\frac{[R]}{V}\right)}
$$

where $[R]$ is molar refraction and $V$, molecular volume. Molar refraction $[R]$ is taken as the sum of atomic refractions.

Intrinsic molecular volume $V_{\text {int }}$ of monomer unit for amorphous polymer can be calculated from the atomic radius and bond length of the constituent atoms based on the method developed by Slonimskii et al. ${ }^{20}$ When an atom $\mathrm{A}$ (atomic radius $R$ ) is bound to atom $\mathrm{A}_{\mathrm{i}}$ (atomic radius $R_{\mathrm{i}}$ ) with bond length $d_{\mathrm{i}}$, the atomic volume $\Delta V(\mathrm{~A})$ of atom $\mathrm{A}$ is given by

$$
\begin{aligned}
\Delta V(\mathrm{~A}) & =(4 / 3) \pi R^{3}-\sum_{i}(1 / 3) \pi h_{i}^{2}\left(3 R-h_{i}\right) \\
h_{i} & \equiv R-\left(R^{2}+d_{i}^{2}-R_{i}^{2}\right) /\left(2 d_{i}\right)
\end{aligned}
$$

If the molecule consists of atoms $\mathrm{A}_{1}-\mathrm{A}_{j}$, the intrinsic molecular volume $V_{\text {int }}$ is given by 


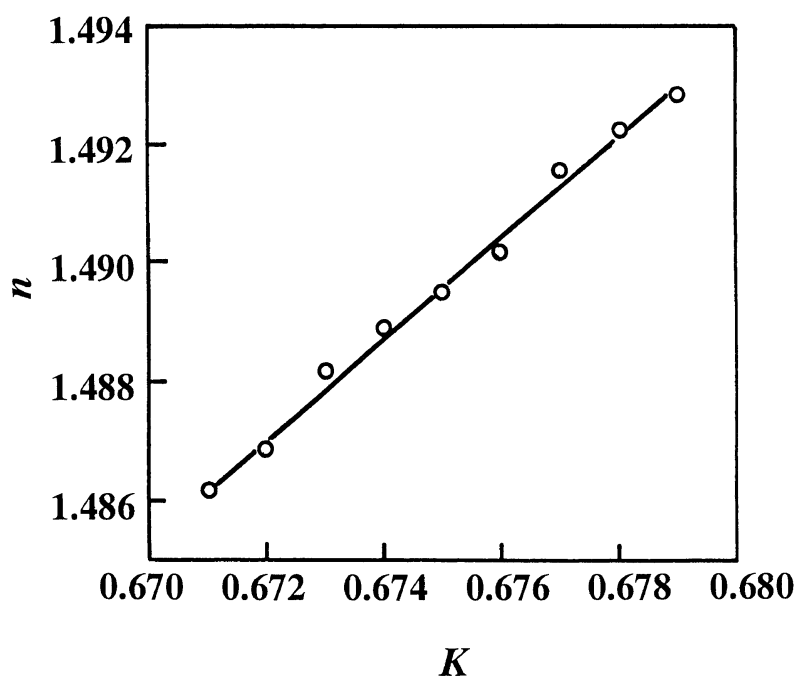

Figure 4. Packing coefficient $(K)$ dependence of the refractive index $(n)$ of PMMA.

$$
V_{\text {int }}=N_{\mathrm{A}} \sum_{j} \Delta V\left(\mathrm{~A}_{j}\right)
$$

where $N_{\mathrm{A}}$ is Avogadro's number. The actual molecular volume $(V)$ of the monomer unit is expressed as

$$
V=\frac{V_{\text {int }}}{K}
$$

where $K$ is the packing coefficient of the molecule. The specific volume $(v)$ of polymer is given by

$$
v=\frac{V_{\text {int }}}{K M_{\mathrm{o}}}
$$

where $M_{0}$ is molecular weight of a monomer unit.

The specific volume of amorphous polymer can be estimated if packing coefficients are given. Packing coefficient $(K)$ dependence of the refractive indices of the compounds can be calculated using above equations. Figure 4 shows refractive indices at $633 \mathrm{~nm}$ for PMMA as function of packing coefficients. Before annealing the refractive index was 1.4888 by a prism coupler, indicating that the packing coefficient $K$ is 0.674 . Figure 5 shows the change of packing coefficient of PMMA with annealing from the data of Figure 3. Figure 6 is the calculated specific volume as function of annealing time for PMMA from the $K$ using eq 11 . The specific volume decreased with aging time. Before aging, specific volume was $0.8561 \mathrm{~cm}^{3} \mathrm{~g}^{-1}$. With increasing time, specific volume decreased, reaching 0.8538 $\mathrm{cm}^{3} \mathrm{~g}^{-1}$ at 70 days. This decrease is caused by densification due to aging below $T_{\mathrm{g}}$, as recognized by enthalpy relaxation in Figure 2.

Prism coupling is convenient and very accurate for detecting volume change to study volume relaxation behaviour.

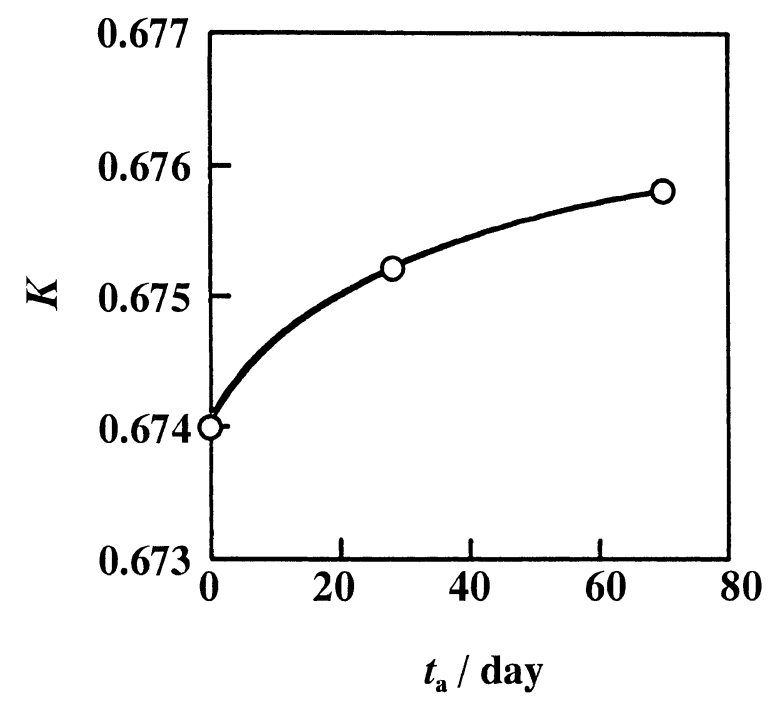

Figure 5. Packing coefficient $(K)$ as function of aging time $\left(t_{\mathrm{a}}\right)$ at $90^{\circ} \mathrm{C}$ for low-loss PMMA glass.

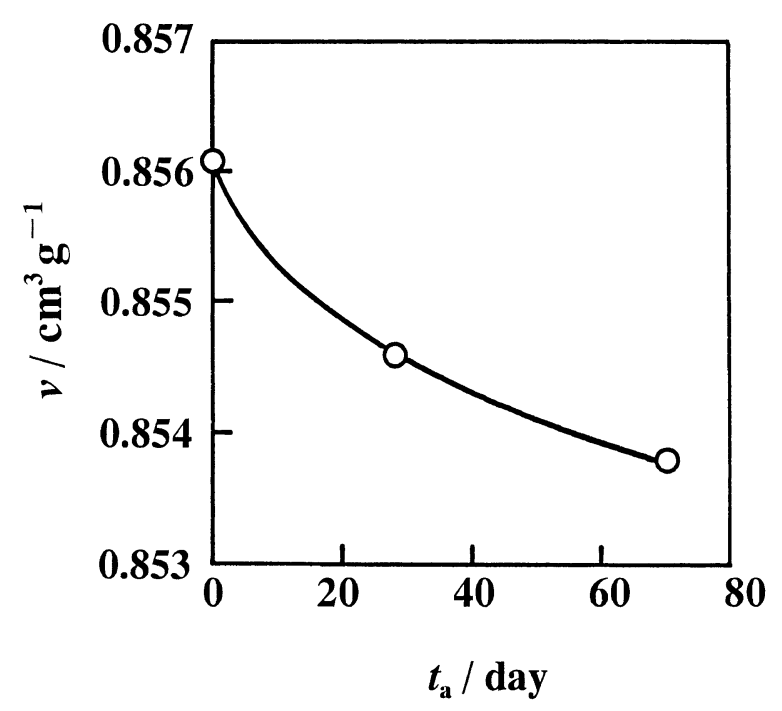

Figure 6. Specific volume $(v)$ as function of aging time $\left(t_{\mathrm{a}}\right)$ at $90^{\circ} \mathrm{C}$ for low-loss PMMA glass.

\section{CONCLUSION}

Structural relaxation of low-loss PMMA glass was studied by optical prism coupling. We measured change of refractive indices of low-loss PMMA glass with annealing at temperatures below $T_{\mathrm{g}}$ and determined volume relaxation by physical aging. Prism coupling is convenient and accurate for detecting volume change.

\section{REFERENCES}

1. L. C. E. Struik, "Physical Aging in Amorphous Polymers and Other Materials", Elsevier, Amsterdam, 1978.

2. A. J. Kovacs, J. Polym. Sci., 30, 131 (1958).

3. R. Greiner and F. R. Schwarzl, Rheol. Acta, 23, 378 (1984). 
4. H. Yoshida and Y. Kobayashi, Polym. J., 14, 925 (1982).

5. J. M. G. Cowie and R. Ferguson, Polymer, 34, 2135 (1993).

6. N. Tanio, H. Kato, Y. Koike, H. E. Bair, S. Matsuoka, and L. L. Blyler, Jr., Polym. J., 30, 56 (1998).

7. Y. Koike, N. Tanio, and Y. Ohtsuka, Macromolecules, 22, 1367 (1989).

8. P. Debye and A. M. Bueche, J. Appl. Phys., 20, 518 (1949).

9. P. Debye, H. R. Anderson, and H. Brumerger, J. Appl. Phys., 28, 679 (1957).

10. P. K. Tien, Appl. Opt., 10, 2395 (1971).

11. T. N. Ding and E. Garmire, Appl. Opt., 22, 3177 (1983).

12. M. J. Bowden, E. A. Chandross, and I. P. Kaminow, Appl.
Opt., 13, 112 (1974).

13. N. Tanio, Y. Koike, and Y. Ohtsuka, Polym. J., 21, 119 (1989).

14. N. Tanio, Y. Koike, and Y. Ohtsuka, Polym. J., 21, 259 (1989).

15. N. Tanio and Y. Koike, Kobunshi Ronbunshu, 53, 682 (1996).

16. A. Einstein, Ann. der Phys., 33, 1275 (1910).

17. E. Muzeau, G. Vigier, and R. Vassoille, J. Non-Cryst. Solids, 172-174, 575 (1994).

18. H. A. Lorentz, Ann. der Phys., 9, 641 (1880).

19. L. V. Lorenz, Ann. der Phys., 11, 70 (1880).

20. G. L. Slonimskii, A. A. Askadskii, and A. I. Kitaigorodskii, Vysokomol. Soedin., Ser. A, 12, 494 (1970). 